\title{
A Modeling Framework for Interplanetary Supply Chains
}

\author{
Erica L. Gralla ${ }^{*}$, Sarah Shull ${ }^{\dagger}$ and Olivier de Weck ${ }^{*}$ \\ Department of Aeronautics \& Astronautics, Massachusetts Institute of Technology, Cambridge MA 02139 \\ Gene Lee ${ }^{\S}$ and Robert Shishko** \\ Jet Propulsion Laboratory, California Institute of Technology, Pasadena CA 91109
}

\begin{abstract}
As NASA looks ahead to next-generation human space exploration, it is essential to consider the cost of operations and support in order to ensure the development of affordable programs. The life-cycle costs of future exploration ventures can be reduced by focusing on the interplanetary logistics strategy. By leveraging proven supply chain management techniques from the military and commercial sectors and applying them to the unique challenges of human space exploration, space logistics can be streamlined, and mission affordability and robustness can be increased. This paper describes a modeling framework for space logistics which enables description, evaluation, and optimization of various types of logistics strategies. The framework is embodied in SpaceNet, a discrete event simulation and optimization software program.
\end{abstract}

\section{Introduction}

Tn order to ensure that NASA's human lunar and Mars exploration program is both affordable and sustainable, it is essential to consider the cost of operations and support. In the past, operations costs have proven to be a major factor in program affordability. Shuttle operations costs are significantly higher than originally envisioned due to changes in the operational architecture of the Shuttle (it flies less frequently than planned, so the fixed operating costs are spread among fewer flights). It is essential to avoid the same types of problems in NASA's next major human space flight venture. Therefore, we look to reducing the life-cycle operations costs for future exploration programs by focusing on the logistics strategy. This paper describes a space logistics model developed by MIT, JPL, and others to understand the supply chain management problem for human lunar and Mars exploration

\section{A. Motivation}

One of the main factors contributing to the operations cost of human lunar and Mars exploration (HLE/HME) architectures is the cost of shipping required cargo and supplies, especially for long-duration missions. It is important that logistics be taken into account at an early stage in the design process, because the exploration architecture and vehicle design can impact logistics-related operations costs. The key question here is how best to ship cargo and supplies for an exploration mission. In order to understand the specific logistics costs associated with various exploration architecture choices, a modeling framework and planning tool for interplanetary space logistics is required.

The other major question requiring an answer is what cargo and supplies should be shipped for an exploration mission. Direct 'value' from exploration is generated not by simply landing on the Moon; rather, NASA must make accomplishments to generate value, such as reaching scientific objectives, testing new technologies, etc. Therefore value is generated by a very small fraction of the launch mass of an exploration mission: propellant makes up approximately $93 \%$ of the launch mass, vehicle dry mass about $6.9 \%$, and everything else only $0.1 \%$, including crew, consumables, spares, exploration items, and other supplies. Therefore, it is extremely critical that we choose

\footnotetext{
* Graduate Research Assistant, MIT Aero/Astro, AIAA Student Member, egralla@mit.edu.

${ }^{\dagger}$ Graduate Research Assistant, MIT Aero/Astro, AIAA Student Member, sshull@mit.edu.

*Associate Professor of Aero/Astro and Engineering Systems, Senior AIAA Member, deweck@mit.edu.

${ }_{\star}^{\S}$ Systems Engineer, JPL Systems Analysis \& Modeling Group, AIAA Member, Gene.Y.Lee@jpl.nasa.gov.

** Principal Systems Engineer/Economist, JPL Systems Analysis \& Modeling Group, AIAA Member, Robert.Shishko@jpl.nasa.gov
} 


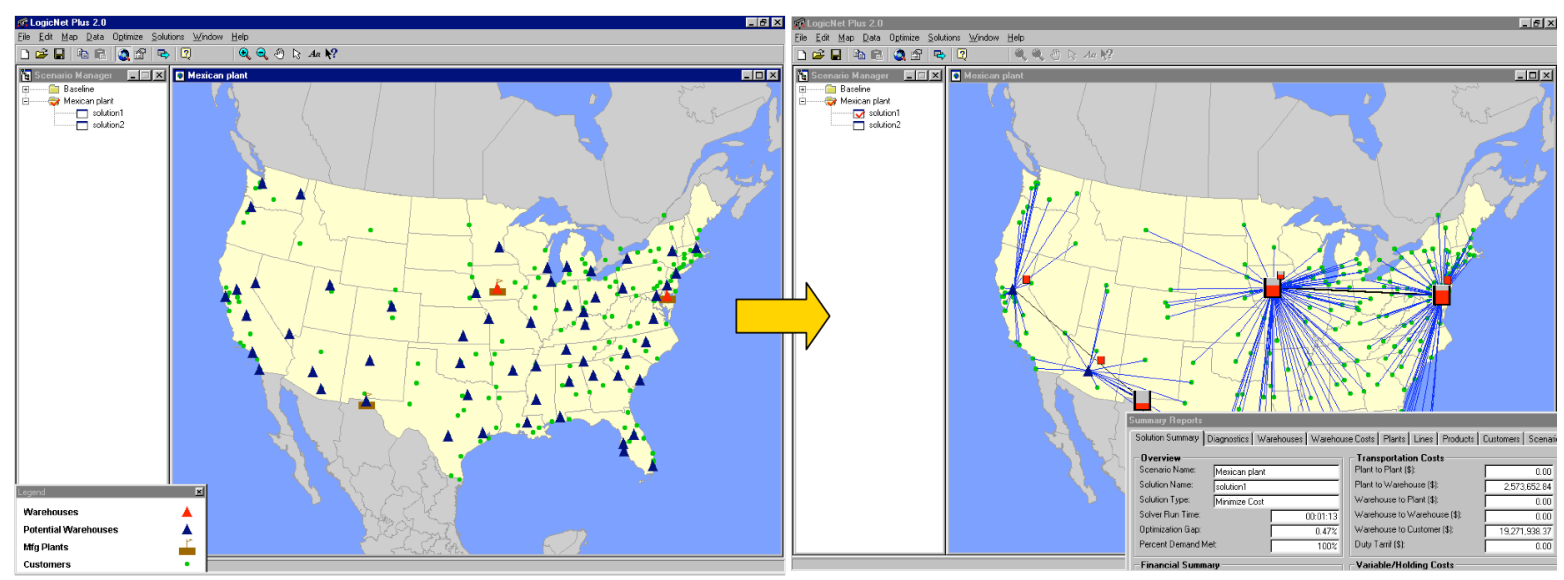

Figure 1. Supply chain network optimization. LogicNet Plus 2.0 commercial supply chain optimization software. The red flags represent factories, the green dots represent retail outlets, and the blue triangles represent potential warehouses. On the left, the problem is shown in the LogicNet software: find the optimal location for warehouses from among all the potential locations. The right-side screenshot shows the solution to the problem, with optimally located warehouses and their shipping networks.

an appropriate set of crew and cargo to maximize the value from that $0.1 \%$ mass fraction. What should be launched, and how often? What is the best way to trade off between consumables (endurance), spares (robustness), and exploration items (value)? These are the types of questions that can be answered only through modeling in detail large sets of future interplanetary logistics scenarios.

\section{B. Background}

The term 'space logistics' means many different things to different people. In this case, we define it loosely as the movement, storage, and tracking of all crew and equipment necessary to carry out an exploration mission or campaign. With this definition, logistics encompasses nearly every aspect of space flight operations (e.g. space flights are movement of crew and cargo), but does not include the detailed design of the vehicles and equipment. In other words, we consider that in a logistics scenario, the vehicle characteristics and available equipment items are given. Such vehicle designs can (and should) be subjected to trade studies, evaluating their relative suitability for logistics. However, by the term space logistics, we imply a more narrowed focus on the operations architecture for transportation and storage of cargo and crew.

Furthermore, we focus this paper on what we term 'interplanetary logistics'. Therefore, we ignore the groundbased component of the logistics architecture (e.g. transportation to and from KSC), and consider only the transportation and storage of items in space, including launch. We acknowledge that ground operations are important and a large contributor to overall costs and must be studied as well. It is also worthwhile to note that we use the terms 'logistics' and 'supply chain management' interchangeably; Simchi-Levi et al. [1] does not find it necessary to define them separately, and we follow the same model here.

Armed with this basic definition of interplanetary logistics, we proceed to discuss how our analysis methods have been based on techniques proven highly successful in commercial and military supply chain management. We then provide background for the study of future logistics architectures by examining past logistics strategies for manned space exploration.

\section{1. 'Terrestrial' Logistics and Supply Chain Management}

Supply chain management in military and commercial enterprises has proven highly successful in recent years, enabling major cost reductions and increases in efficiency. Generally these gains have been achieved by simultaneously reducing shipping costs, reducing inventory holding costs while increasing service levels. We term these efforts 'terrestrial logistics and supply chain management', meaning supply chain management techniques developed for the commercial and military sectors. Terrestrial logistics and supply chain management is a highlydeveloped field; techniques for efficient supply chain management have been proven very effective in the business case, affording enterprises a competitive advantage [2]. The wealth of information on this area can be applied to the interplanetary problem in order to develop a model for understanding space logistics. 
Three key concepts have enabled many of the recent advances in terrestrial supply chain management, and can also be applied to space exploration:

1. Network-wide view rather than location-specific. In other words, replacing local decisions with global optimization.

2. Ability to manage risk effectively through push-pull and risk pooling.

3. Lean design, or product and system strategies that take into account life cycle cost drivers.

The first concept leads to supply chain design methods based on networks and optimization. For example, Figure 1 shows the process of selecting optimal warehouse locations using a software package called LogicNet. Based on the given locations of factories (red squares) and retail outlets (green dots), a large set of potential warehouse locations (blue triangles) can be evaluated to find the optimal warehouse placement for the minimization of transportation costs and shipping times, for example. Such network design techniques can also be used to find globally optimal logistics strategies for space exploration.

The second concept centers around the idea of push and pull strategies. Companies can reduce inventory and costs by operating a 'pull' supply chain in which products are only shipped (or assembled) when they are ordered, for example. On the other hand, better customer service results from 'push' supply chains which make products available before an explicit demand occurs. Combining these two strategies into a 'push-pull' supply chain, where some inventory (generally lower levels of the product decomposition) is pushed while other inventory is pulled, explores the trade-off between cost and customer service (service level). In the interplanetary realm, the same concepts can be used. Because Mars is so far away, the push strategy appears to be the only logical choice, as demands arising on Mars mid-mission cannot be satisfied due to periodic (2 1/7 year) launch windows. On the other hand, the International Space Station ships spares for large orbital replacement units (ORUs) such as control moment gyros only when needed - utilizing the 'pull' strategy. Moreover, some supplies are always carried along with the crew. Combining these ideas could generate new optimal strategies for future space missions.

Finally, the third concept deals with ideas that aerospace systems engineers are already familiar with: designing systems that take into account life cycle costs. All three of these concepts are relevant to logistics planning for space exploration; however, several significant distinctions arise in the realm of space missions. First, the significance of fuel (propellant) in space missions is much higher than in commercial enterprises. Fuel is not explicitly accounted for in commercial supply chains, but its mass is of paramount importance in space missions, consuming most of the mass of a spacecraft in many cases. Trucks can usually be easily refueled en-route to their destinations; spacecraft cannot (at present). The second distinction is driven by the time required to traverse the vast distances in space: supplies can gain or lose mass en-route to the destination (as food is eaten and waste is produced). In commercial supply chains, the mass and volume of supplies are generally considered static during transportation. Finally, a third difference arises in the nature of the transportation routes themselves: the costs of traversing roads and waterways do not change with time, but available trajectories in space vary with the movements of the planets (e.g. practical Mars trajectories are only available every two + years). The chart in Figure 2 shows several of the analogues between commercial and space logistics. With these types of similarities and distinctions identified, we can build on the rich heritage of success in private supply chain management to improve logistics for space exploration.

\begin{tabular}{|c|c|c|c|}
\hline & & Terrestrial Commercial & Space Exploration \\
\hline \multirow{2}{*}{$\begin{array}{l}\text { Concept } 1 \\
\text { Networks }\end{array}$} & Nodes & $\begin{array}{l}\text { Suppliers, manufacturers, } \\
\text { distributors, retailers }\end{array}$ & $\begin{array}{l}\text { Launch sites, orbital } \\
\text { nodes/depots, surface ops }\end{array}$ \\
\hline & Arcs & $\begin{array}{l}\text { Transportation links: truck, } \\
\text { rail, air, barge, cargo ship }\end{array}$ & $\begin{array}{l}\text { Propulsive elements: launch, } \\
\text { chemical/electric rockets }\end{array}$ \\
\hline \multirow{2}{*}{$\begin{array}{l}\text { Concept } 2 \\
\text { Push-Pull }\end{array}$} & Supply Items & SKUs (Stock Keeping Units) & $\begin{array}{l}\text { Consumables, spares, } \\
\text { exploration items, } \ldots\end{array}$ \\
\hline & Demand & $\begin{array}{l}\text { Generated by customers } \\
\text { (online \& retail stores) }\end{array}$ & $\begin{array}{l}\text { During in-space transit, } \\
\text { while exploring on surface }\end{array}$ \\
\hline $\begin{array}{l}\text { Concept } 3 \\
\text { Lean }\end{array}$ & Elements & $\begin{array}{l}\text { Modular, easily upgradeable } \\
\text { products }\end{array}$ & $\begin{array}{l}\text { Modular, easily maintainable } \\
\text { vehicles }\end{array}$ \\
\hline
\end{tabular}

Figure 2. Terrestrial analogies to space logistics. Based on the three key concepts derived from commercial supply chain management, listed at left, we identify the building blocks for an efficient supply chain: nodes, arcs, supply items, demand, and elements. Examples of each for commercial and space logistics are given. 

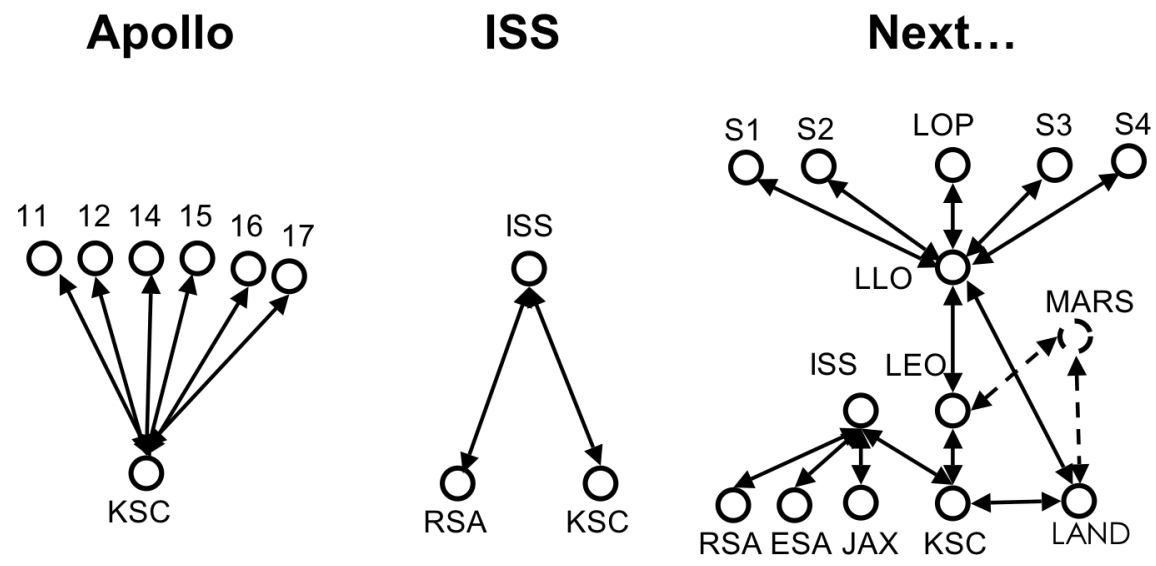

Figure 3. Past space mission supply chains. The Apollo program (left) operated with one Earth-based node at KSC from which six independent missions were sent to six lunar surface sites. In ISS (center), on the other hand, two Earth-based nodes supply one in-space node (RSA refers to the Russian launch site). In the future (right), several Earth-based nodes may support operations of both ISS and exploration missions to various bases and sites on the Moon or Mars.

\section{Space Exploration Logistics: Past, Present, and Future}

Past human space exploration programs have followed different types of logistics paradigms. Under the Apollo program, six missions to the lunar surface were conducted between 1969 and 1972. Each mission was selfcontained; in other words, no space logistics network existed to support each mission. Instead, all the supplies were carried with the astronauts to their destinations. Forecasts predicted the number and type of supplies that would be needed on the lunar surface to support the short-term lunar missions. This logistics strategy can be termed the "backpack model" or "carry along" because of its resemblance to hikers carrying all their equipment in backpacks and discarding or consuming supplies along the way. This type of strategy is clearly practical and perhaps even optimal for short-term missions like those of the Apollo program.

On the other hand, for the International Space Station (ISS) program, a "backpack model" logistics strategy was extremely impractical because of the long duration of the mission: supplies for several years of operations could not be stored on the station. Instead, the ISS logistics strategy is based on regular resupply flights by various vehicles, including the American Space Shuttle and the Russian Progress and Soyuz vehicles. The number and type of supplies shipped is generally based on the actual demand generated on the Space Station, rather than forecasts predicting supply requirements. This strategy can be termed "scheduled resupply," the same strategy used by people the world over who replenish their pantries from the grocery store once a week. This type of strategy is appropriate for long-term missions located relatively near a resupply source (i.e. grocery store). Note that in the case of ISS the resupply schedule is generally fixed, while the exact manifest of what is being resupplied is not.

Figure 3 depicts the basic networks behind each of these logistics paradigms, and also includes the growing network that may be needed to support the next-generation space exploration programs now in the planning stages. Relatively simple logistics strategies functioned well for the two major programs that have been operated to date, but the next-generation network appears much more complex. What is the best logistics paradigm for nextgeneration missions? We hypothesize that it lies somewhere on the spectrum between the "backpack" and "resupply" models, perhaps including the more specialized idea of "pre-positioning" supplies for future use, but it is unclear exactly what combination of these strategies will provide the most affordable, most robust supply chain for next-generation programs.

\section{Goals}

Because of the recognized need to reduce lifecycle operations costs for future programs, and the mounting complexity of supplying missions to distant planetary bases, logistics operations for next-generation spaceflight must be streamlined. We seek to investigate various logistics paradigms and develop appropriate supply chain management strategies for Moon and Mars exploration. The following are key logistics questions for exploration, which serve to guide this research and whose answers are sought as part of this study: 
- What is the most appropriate supply chain strategy for Earth-Moon-Mars exploration? Does it incorporate pre-positioning, the backpack model, scheduled resupply, or hybrids of these ideas?

- For what, when, and where is it cost-effective to establish an on-orbit or surface depot? For spares, consumables, and/or fuel? Before, during, or after the buildup of a lunar outpost? In LEO, LLO, or at a Lagrange point?

- How do uncertainties impact logistics and exploration capability? This includes component failures, launch failures and delays, and stochastic consumption rates.

- What is the net effect of in-situ resource utilization (ISRU) on total launch mass, system cost, and campaign risk?

In order to answer these and other similar questions, we must be able to describe and calculate the variations in logistics strategies quantitatively, which has not been extensively studied in the area of space exploration. We must be able to model different types of supply chains in order to calculate their effectiveness and compare various paradigms. In order to accomplish this, we borrow supply chain modeling and analysis techniques from the private and military sectors, and determine how to apply them to the unique challenges of space exploration logistics.

As part of a larger project, we have studied terrestrial supply chains that are particularly analogous to space exploration [3] and investigated several databases to cull essential 'lessons learned' from past NASA logistics approaches [4]. In this paper, we describe the modeling framework we have developed for describing, modeling, and evaluating logistics architectures, as well as the implementation of this modeling framework in a tool called SpaceNet - a simulation, planning, and optimization tool for space logistics.

\section{Modeling Framework for Interplanetary Logistics}

\section{A. Overview and Challenges}

One of the major challenges in the development of a space logistics model is defining the model components. Interplanetary logistics has not been previously modeled, so the scope of such a model must be defined. The term 'logistics' encompasses a wide area of space flight planning. Obviously, logistics describes the movement of crew ${ }^{*}$, cargo, and vehicles from one place to another - such as from the Earth to the Moon. However, there are multiple dimensions to this problem; for example, moving cargo from LEO to low Lunar orbit (LLO) requires a very different transport vehicle from landing on the lunar surface. Launch also requires a distinctly different vehicle. Furthermore, cargo shipments can be accomplished in many subtly different ways: pre-positioning, carry-along, and staging 'warehouses' are all variations on shipment strategy. These distinctions and more must be accounted for in the model.

Optimal shipment strategies depend on the amount and type of cargo to be shipped. Given a quantity of cargo, a source and destination, and transportation alternatives, it is relatively straightforward (although not easy in the interplanetary case) to determine a good shipment strategy. However, the exact destinations and quantity of cargo are currently unknown for NASA's HLE/HME missions. Thus, any logistics modeling framework must also incorporate a model of demand for each mission scenario. Cargo of various types may also require different handling, so demand must be understood at the level of a supply 'class' - a general category of supply (such as food, spares, or science equipment). Modeling demand can be straightforward in certain cases (e.g. food consumption) but very difficult in others (e.g. spares).

In order to keep track of the demand levels at various locations and allocate shipments to fill them, an information architecture must be designed. Information architecture is a major challenge even for terrestrial logistics today, as new technologies such as radio-frequency identification (RFID) come on the scene. We take all these options into account in designing a relational database that tracks demand and shipments of cargo by supply class (the database is discussed in a companion paper [5]).

Finally, we must model the entire logistics scenario end-to-end for a complete series of missions. This requires a simulation capability, which links all the previously discussed components into a cohesive model. With the simulation in place, trade studies can be run to compare the effects of various logistics strategies. In addition, an optimization component can be added to identify other good strategies for each mission scenario.

To summarize, we have identified a number of components required for an interplanetary logistics model:

\section{- Movement or shipment of crew, cargo, and vehicles}

\footnotetext{
* The focus is on modeling crewed (manned) space exploration missions; unmanned (robotic) flights can be included as a subset.
} 
- Demand by supply class

- Information architecture

- Simulation

- Optimization

The challenge of integrating these components into a cohesive end-to-end logistics and operations model is discussed in the next sections.

First, we describe the basic building blocks of our modeling framework (nodes, elements, and supplies), along with two concepts which enable us to tie these together: the time-expanded network and processes for movement through the network. Collectively, this framework allows us to describe and model both the demand and the movement of items in the logistics scenario (the first two bullets above). Finally, we describe the remaining layers which enable the effective utilization of this modeling framework: the ability to simulate and evaluate various architectures, and even to apply optimization techniques.

\section{B. Building Blocks: Nodes, Elements, and Supplies}

This section describes the basic building blocks in our modeling framework: nodes, supplies, and elements. They are derived from terrestrial supply chain management and from past practices in space logistics.

1. Nodes

Nodes are spatial locations in the solar system. Contrary to some usages of the term, the existence of a node does not necessarily indicate that a facility exists at that location or that a node is ever used or visited. A node is simply a way to refer to locations in space. Nodes can be of three basic types:

- $\quad$ Surface nodes

- Orbital nodes

- Lagrangian nodes

Surface nodes are fairly straightforward. They exist on the surface of a central body such as the Earth, the Moon, or Mars, and they are further characterized by their latitude and longitude on that central body. Examples of surface nodes include Kennedy Space Center and the Apollo 11 landing site at Mare Tranquilitatis.

Orbital nodes are also characterized by their central body (e.g. Earth, Moon, Mars, or Sun), as well as other characteristics describing the orbit itself: apoapsis, periapsis, and inclination". Therefore, the ISS orbit could be an orbital node located around Earth at an altitude of $400 \mathrm{~km}$ and an inclination of 51.6 degrees. Similarly, a low lunar orbit (LLO) is a commonly used orbital node in lunar exploration missions.

Lagrangian nodes are located at any of the Lagrange points in the solar system. They are characterized by the two bodies and the number of the Lagrange point. One commonly considered Lagrange point is the Earth-Moon L1 point, which lies between the Earth and the Moon at the point where the two bodies' gravitational pulls are balanced.

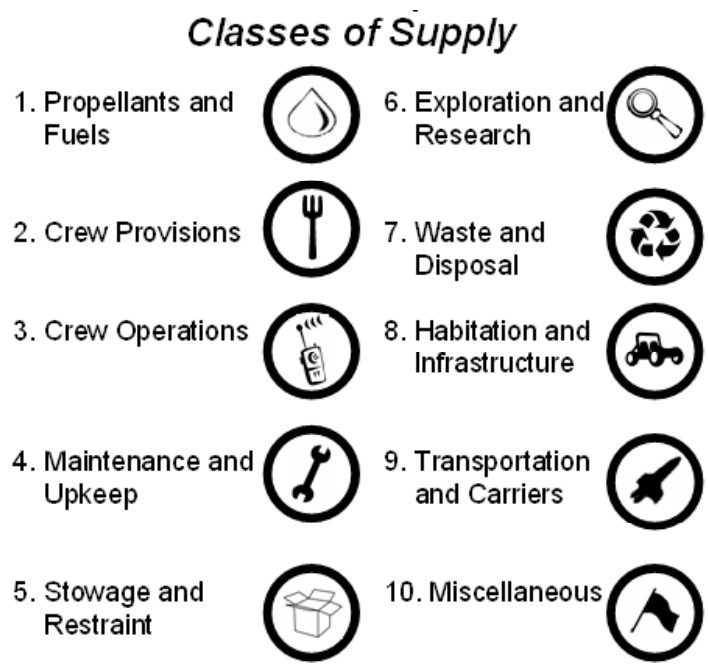

Figure 4. Functional classes of supply. This set of ten classes of supply is based on the essential functions of a planetary base. Categories such as Class 2 include both the provisions themselves and the equipment required to utilize them - heating implements, water storage devices, etc.

\footnotetext{
* At the level of abstraction of SpaceNet only those orbital elements that are energetically relevant are modeled explicitly.
} 
We reiterate that labeling a location as a node does not necessarily mean a permanent facility exists at that location. Rather, it means that some part of the logistics architecture for a space mission might make use of that location as a transit or waiting point. For example, if a spacecraft is launched from KSC to LEO, then propelled toward lunar equatorial orbit, it has passed through one surface node and two orbital nodes. The nomenclature developed around nodes allows us to build up a potential transportation network and thus to formalize description of logistics architectures.

\section{Supplies}

Supplies are the items that move through the network, from node to node. Generally, supplies should include all the items needed at the planetary base, or during the journeys to and from the base. Examples include consumables, science equipment, surface vehicles, and spares. In order to track and to model the extraordinary variety of supplies that could be required, they must be classified into larger categories. This study spent a great deal of effort analyzing various

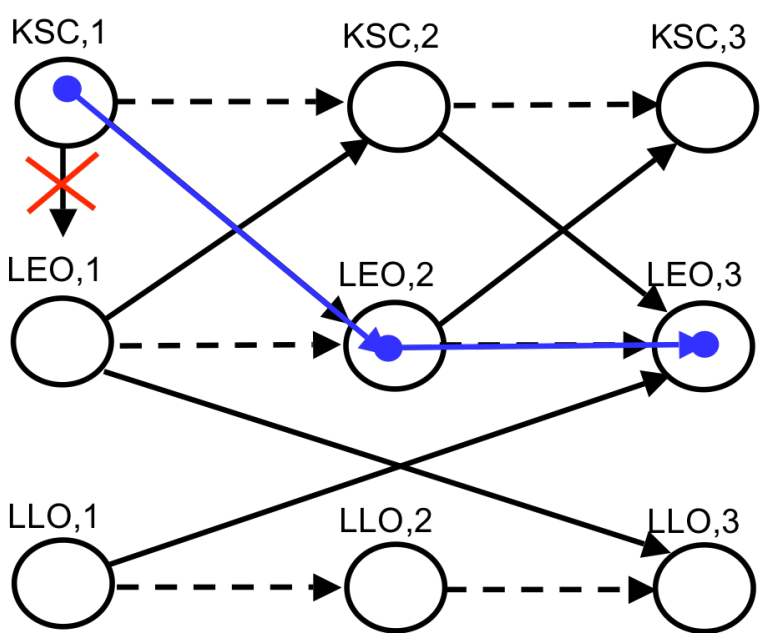

Figure 5. Time-expanded network example. The static nodes KSC, $L E O$, and LLO are copied at each of three time steps, and the allowable arcs (transitions) between them are shown as arrows. Dashed horizontal arrows indicate waiting arcs. ways to classify supplies (see [5]), and concluded that the best method was to develop a set of functional classes of supply, organized regardless of material or owner. The classes are therefore based on the essential functions of a planetary base, or the tasks that need to be accomplished, such as research, habitation, transportation, etc. The final set of ten classes of supply (CoS) is shown in Figure 4. (NASA's CCART classification system - in use for ISS logistics - was evaluated for use in this context, but it was occasionally inconsistent and was missing a number of categories required for surface exploration.)

Most of the classes are self-explanatory. Class 4 includes maintenance equipment as well as spares for all types of equipment. Class 3 includes general base operations equipment such as communications, while Class 6 describes equipment specialized for research and exploration. Class 2 includes both crew provisions and support equipment such as eating utensils and food preparation devices. Class 9 includes the vehicles used for transportation in space.

These classes of supply can then form the basis for the modeling of supply items. Recall that the impetus behind the development of these supply classes was the need for a manageable modeling framework for supplies moving through a transportation network. With these ten supply classes we can model demand for various types of items at the supply class level. In addition, we can more easily simulate and track the movement of these aggregate supply items through the transportation network, using a unified relational database for exploration [5]. For example, a planetary base might require 10 units of crew provisions, rather than certain amounts of water, dried food, drink mix, eating utensils, etc. With these classes of supply, the modeling problem can be reduced to a manageable size."

\section{Elements}

Elements are defined as the indivisible physical objects that travel through the network and (in general) can hold or transport supplies. Most elements are what we generally think of as "vehicles" - the crew exploration vehicle (CEV), propulsion stages, etc. Here, we also include other major end items such as surface habitats and pressurized rovers. Elements, then, are characterized by a wide set of characteristics: they can

- hold other supply items (e.g. fuel or cargo)

- be propulsive or non-propulsive

- carry crew or not carry crew

- be launched from Earth

\footnotetext{
* For more detailed demand forecasting, a total of 44 sub-classes of supply were developed, but these are subsequently aggregated into the ten classes shown in Fig. 4.
} 
- be reused, refueled, disposed of (staged), pre-deployed

- be "docked" with other elements to form a (temporary) stack

In general, an element has defined capacities for three types of items: crew, cargo, and propellant. These capacities determine what types of supplies can be assigned to that element for transport, and whether the element is propulsive. Thus, elements can transport supplies and crew between the various nodes of the transportation network.

\section{Tying it Together: Network and Processes}

With the preceding definitions of nodes, supplies, and elements, we have defined the basic building blocks of a modeling framework for space logistics. We can create a network of nodes, and define elements capable of traversing that network between nodes, carry supplies. Two remaining concepts are needed to tie these ideas together: the time-expanded network (to account for changes in trajectories over time) and processes that describe how elements and supplies move through the network.

\section{Time-Expanded Network}

A time-expanded network is a concept that builds on the idea of a static network. We have discussed the creation of a static network based on nodes like KSC, LEO, and LLO. We can take that static network and expand it in time, to account for changes in the network over time.

Refer to Figure 5 for a simple example of a time expanded network. The static network is made up of the three nodes along the left-hand side of the figure, labeled 'KSC,1', 'LEO,1', and 'LLO,1'. We then use a time step $\Delta t$ and expand these three nodes forward in time: at time step two, therefore, we copy each of the static nodes, so that the middle column in Figure 5 is labeled 'KSC,2', 'LEO,2', and 'LLO,2'. We copy these nodes again for time step three, creating the right-most column. The next step is to define the allowable transitions - called arcs - between the nodes. It is always possible to remain or wait at a given node through the next time step. Therefore we can define all of the horizontal arcs (represented by dashed arrows) shown in Figure 5. Next, we look at transitions from KSC to LEO. The vertical arrow from ' $\mathrm{KSC}, 1$ ' to 'LEO,1' is crossed out because it is impossible to make an instantaneous transition from KSC to LEO. In this example, it takes one time step to make that transition, so arrows are drawn from ' $\mathrm{KSC}, 1$ ' to ' $\mathrm{LEO}, 2$ ' ' and ' $\mathrm{KSC}, 2$ ' to 'LEO,3'. The reverse arcs from LEO to KSC are also added. Finally, the transition from LEO to LLO (in this notional example) takes longer: two times steps are required, so the arcs are

\section{Wait}

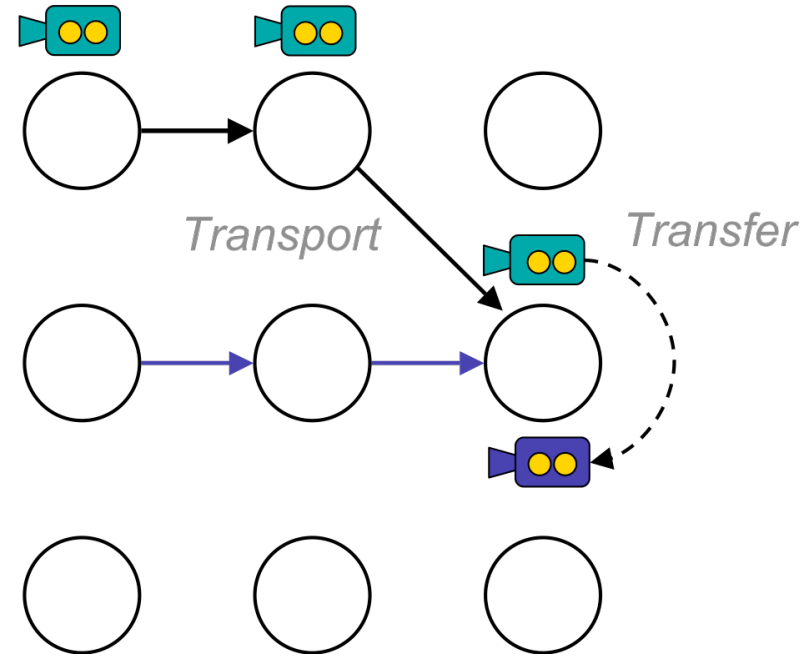

Figure 6. Processes for moving through the network. The wait process describes an element waiting at a given node. The transport process moves an element to a new node, and the transfer process moves crew and/or supplies to a new element at the same node. drawn as shown in Figure 5. This completes the definition of the time-expanded network in our simple example: we have defined time-expanded nodes, waiting arcs, and feasible transport arcs (filtered by the astrodynamic constraints). Now, we can define paths through the network; Figure 5 highlights in blue a path through KSC,1 to LEO,2 to LEO,3 (a transfer from KSC to LEO and a wait at LEO).

Notice that arcs are only defined in the forward direction, because it is impossible to traverse backward in time (non-causal paths are forbidden). Note also that while this network is relatively simple, the construction of such a network is nontrivial for large time horizons or large static networks. A realistic time expanded network with a 3year scenario (about 1000 days), a time step of 1 Earth day and 10 nodes will have 10,000 nodes once expanded in time.The advantages of this type of network construction are that it makes time explicit and enables simulation and optimization of time-varying transportation problems, such as the launch windows to Mars. 


\section{Processes}

With the time-expanded network defined, the only remaining step is to describe how elements and supplies are allowed to move among the nodes of the time-expanded network. There are three essential processes that describe this movement:

$\begin{array}{ll}\text { - } & \text { Waiting } \\ \text { - } & \text { Transporting } \\ \text { - } & \text { Transferring }\end{array}$ remain at the same node move to a new node along an allowable arc transfer crew and/or supplies to a different element

These three processes are illustrated in Figure 6. At this point, with the building blocks, the network, and the processes defined, we can model the flow of supplies, elements, and crew through the logistics network.

\section{Wrapping it Up: Optimization, Simulation, and Evaluation}

The final step in building an effective modeling framework is to add wrappers that allow logistics architectures described by the model to be created, visualized, and evaluated. In the following sections, we first describe the development of an optimization capability which can return optimal logistics architectures within the modeling framwork described above, based on a given demand scenario. Alternatively, logistics architectures could be created by hand. Second, we describe the essential simulation capability which takes a described architecture and simulates it to ensure demands are met, transport arcs have sufficient fuel, etc. Finally, the scenario can be visualized and evaluated using various types of tools built over the previously defined modeling framework.

\section{Optimization}

In some cases, we envision that pre-defined architectures will be evaluated against one another, resulting in a logistics trade study. In other cases, however, the best approach would be to calculate the demand for various supplies at a lunar base for example, and ask the software to find the optimal logistics architecture to supply that mission (or series of missions). This is the goal of the optimization layer. The modeling framework was specifically built to allow for optimization. The description of this capability is outside the scope of this paper; for further information refer to [6] and [7].

\section{Simulation}

A logistics architecture can be described using the modeling elements discussed above. However, in order to determine the effectiveness of the architecture, it must be simulated so that it can be evaluated in relation to others. The simulation ties together all other components of the modeling framework, taking the mission scenario as an input and producing the output information to fully describe and evaluate the mission scenario.

\section{Evaluation: Measures of Effectiveness}

The final step is to process the simulation output in such a way that architectures can be evaluated and compared. In the section describing our implementation, below, we go into detail on the visualization and data output methods developed. Here, we discuss the metrics (termed measures of effectiveness or MOEs) developed hand-in-hand with our modeling framework to enable the comparative evaluation of logistics architectures.

The MOEs defined here provide a quantitative way to evaluate specific space exploration scenarios and interplanetary supply chains in general. While we believe that these MOEs are important and relevant for space exploration logistics, they are only proxy metrics for comparative purposes, not for absolute forecasting. For example, the current benefit metrics do not take into account benefits derived from the presence of robots on planetary surfaces or from orbiting spacecraft.

The value of planetary space exploration research comes primarily from healthy, motivated, and qualified explorers and scientists being able to spend a certain amount of time at one or more planetary surface locations (nodes). To first order, the exploration benefit should scale linearly with both the number of people (crew size) as well as the duration of their stay. In order to do productive research, the crew needs to have with them specific exploration equipment and scientific instruments, such as cameras, rock hammers and so forth. Also, surface infrastructure items (habitation facilities, surface mobility systems, etc.) act as enablers and multipliers for exploration productivity. Thus we define the concept of "exploration mass," which includes both science equipment and infrastructure mass. Based on these ideas, we propose four basic logistics performance measures of effectiveness: 
- Crew Surface Days [crew-days]: The total number of crew-days over all surface nodes for the entire scenario.

- Exploration Mass Delivered [kg]: The total mass of exploration items and surface infrastructure delivered over all surface nodes for the entire scenario.

- Total Launch Mass [kg]: The total launch mass (including crew, elements, and all other COS) for the entire scenario.

- Upmass Capacity Utilization [0,1]: The fraction of the upmass capacity (from Earth) used by all COS (excluding crew, propellants, and elements) for the entire scenario. Ideally, this should always equal 1.

The next set of MOEs attempts to capture the exploration capability of a given logistics architecture. To first order, the exploration capability is the amount of time the crew gets to spend doing exploration and research at a surface node, multiplied by the amount of total exploration mass they have to do the job at each node visited during the scenario. The amount of time the crew can spend doing exploration and research is limited by a number of factors. These sources of crew non-availability include: housekeeping activities, maintenance and repair, in-situ crew activity planning/scheduling, medical, EVA preparation, and physiological (exercise, sleep/rest, eating). In general, the fraction of non-available crew-hours may vary with the size of the crew at the surface node and the length of the surface stay. The following set of 'exploration capability' MOEs captures these ideas:

- Exploration Capability [kg*crew-days]: The dot product of crew surface days and exploration mass (exploration items plus surface infrastructure) over all surface nodes for the entire scenario. Therefore, exploration capability is only accrued when crew and exploration mass are present at a surface node together (co-located).

- Relative Exploration Capability [0, inf): A normalized measure of exploration logistics efficiency, which measures the amount of productive exploration that can be done for each kilogram of mass launched from the Earth's surface, relative to Apollo 17 . $^{*}$ Technically, the calculation depends on the exploration capability divisia index [8] with Apollo 17 normalization. Practically speaking, the calculation resembles Eq. 1.

$$
\text { REC }_{b}=\frac{\text { ExplorationCapability }_{b} / \text { TotalLaunchMass }_{b}}{\text { ExplorationCapability }_{\text {Apollo17 }} / \text { TotalLaunchMass }_{\text {Apollo17 }}}
$$

The relative exploration capability (REC) is a powerful metric for supply chain comparison. A supply chain with a relative exploration capability greater than unity would appear to be more efficient than Apollo 17 because more exploration capability is provided for each unit of mass launched from Earth. The REC is influenced by a number of factors such as the chosen mission/transportation architecture, the use of various propulsion technologies, implemented supply chain strategies such as on-orbit depots, and the application of ISRU technologies. For example, the effects of ISRU can be captured: if no ISRU is applied, a certain amount of consumables directly contribute to the total launch masses in Eq. 1. If ISRU is used, the consumables mass over the entire scenario might be reduced, but the upfront mass penalty for transporting ISRU equipment to a node in the first place would also be captured. Whether or not an investment in ISRU is worthwhile for a particular scenario can then be assessed by comparing the REC of both alternatives.

We attempt to capture the relative cost and risk of various logistics scenarios through two relatively simple measures of effectiveness (below). Note that these are by no means absolute measures of the cost or risk of any given scenario, but should serve to show which scenarios are more or less costly/risky than others.

- Relative Scenario Cost [0, inf): The weighted sum of the total launch mass and the number of element active days for the entire scenario, using weights such that relative scenario cost for Apollo 17 is unity.

- Scenario Risk [0,1]: Defined as 1 minus the probability that there are no failures in all launches, rendezvous-and-dockings, and landing for the entire scenario.

\footnotetext{
* Apollo 17 is used as the reference case because it can be argued that of all the Apollo lunar surface missions, Apollo 17 was the most productive in terms of exploration and science and also the one that came closest to approaching the constraints imposed by flight hardware elements and operational capabilities at that time.
} 
This section has provided an overview of the measures of effectiveness that make up the evaluation portion of this modeling framework. Specific equations and significantly more detail can be found in [9].

\section{E. Modeling Framework Summary}

In this section, we have defined a complete modeling framework to enable the description and evaluation of space logistics architectures. The basic building blocks are the static network of nodes, the supply items required for exploration, and the elements that move through the network and carry the supply items. These building blocks are tied together with a time-expanded network and a set of processes describing how elements and supplies move through the network. With these five concepts, almost any logistics architecture can be described. Finally, a number of wrapper layers can be added to this basic framework, including optimization and simulation capabilities. In addition, a set of metrics has been developed to enable comparisons between architectures described with this framework.

\section{Model Implementation: SpaceNet}

This section describes an implementation of our modeling framework in the form of a logistics planning tool called SpaceNet. SpaceNet has been used for trade studies in support of NASA's Constellation program.

\section{A. SpaceNet Overview}

SpaceNet is a computation environment for modeling exploration from a logistics perspective. It includes discrete event simulation at the individual mission level (e.g. sortie, pre-deploy, or resupply) or at the campaign level (i.e. set of missions). It also allows for the evaluation of manually generated exploration scenarios with respect to measures of effectiveness and feasibility, as well as the visualization of the flow of elements and supply items through the interplanetary supply chain. Finally, it includes an optimization capability and acts as a software tool to support trade studies and architecture analyses.

A diverse user base is envisioned, including mission and system architects, mission planners and logisticians, and even operations personnel in future years. The goals for the tool are to support both short- and long-term architectural and operational decisions, such as:

- What effect will vehicle (element) design decisions have on future NASA operations and lifecycle costs?

- Should a staging area or depot be constructed? Where?

- Are in-space refueling and ISRU helpful in improving performance?

- Is it better to have cargo vehicles that carry small resupply loads or a few large pre-deploy or resupply flights?

SpaceNet is built on the modeling framework described earlier in this paper. Missions are modeled on a network of nodes and arcs, with elements carrying supplies through the network. Demand is generated based on the required

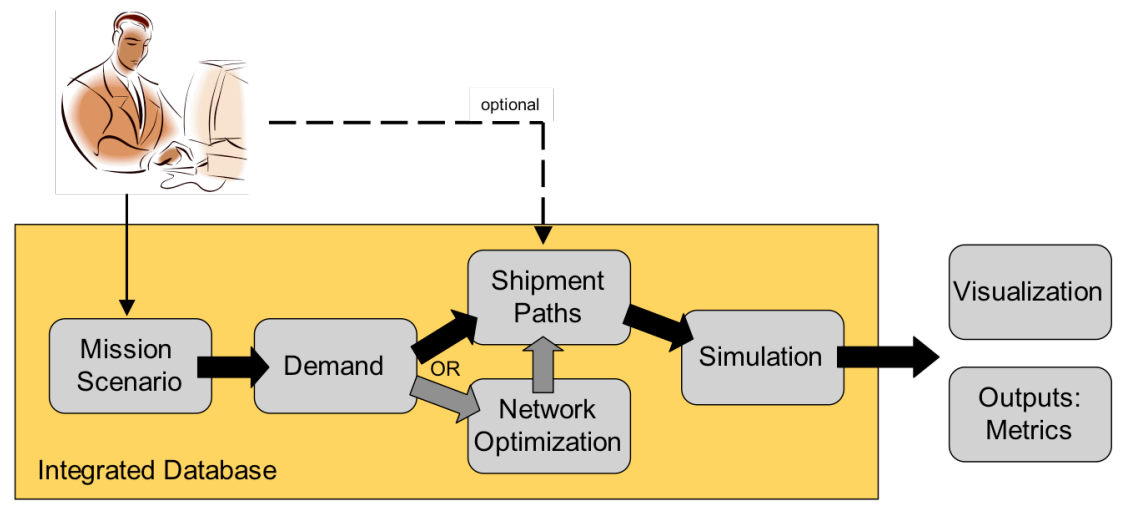

Figure 7. SpaceNet Architecture. The diagram illustrates the architecture and usage concept for SpaceNet. The user defines the mission scenario, which generates demand. The user can then choose to define shipment paths or ask the optimization to accomplish this step. After simulation, the outputs and visualization are created. 
length of surface stay and in-space transportation, and a simulation ensures that demands are met for a given scenario (undersupply situations are explicitly flagged as error conditions). The following sections provide more information.

\section{B. SpaceNet Concept and Usage}

With the basic modeling framework described above, the most straightforward introduction to SpaceNet is to explain the manner in which the tool is used. Figure 7 provides an overview of the architecture and usage concept. First, a mission (usually at a surface node) is defined, then demand is generated based on that mission. Demand must then be satisfied by defining the paths through the network (these can be defined by hand or through optimization) that will transport the required supplies. Finally, with the logistics architecture thus defined, the scenario can be simulated and analyzed.

\section{Define a Mission}

The first step is to define the 'nodal mission' - usually missions take place at surface nodes but occasionally at places like the ISS. Mission attributes must be specified, including the mission location, the crew size and duration, the exploration items required, and the infrastructure (e.g. habitats) that must be delivered. In addition, the entire transportation network can be defined here by choosing the relevant nodes (e.g. Earth, Moon, LEO, LLO, lunar surface) and the elements to be considered as part of this scenario (e.g. CEV, LSAM, etc.). For example, a Constellation sortie might be defined as taking place at Mare Tranquilitatis with a crew of 4 for 7 days, utilizing the CEV, LSAM, EDS and both Ares launchers (CLV and CaLV), and requiring no supporting infrastructure. The nodes in the network are for example KSC, LEO, LLO, the lunar surface base, and Edwards AFB (land landing).

\section{Generate Demand}

Demand generation is largely automated by SpaceNet. The required types and amounts of each of the relevant supply classes is estimated based on the surface mission and transportation arcs (if defined). The parameters for this demand model can be edited by the user, but default values are provided. The model for six of the ten supply classes (fuels, habitation/infrastructure, elements, and 'Miscellaneous' supplies are handled separately) is based on an extensive database of supply items and spares, and each of these models can be upgraded as needed.

\section{Satisfy Demand: Paths and Optimization}

The third step is to satisfy the demand generated in the previous step (and that incurred by transportation time) by defining how crew and supplies will move through the network to accomplish the mission. This step essentially requires the description of the transportation architecture. Optimization can be utilized so that the software suggests an appropriate transportation architecture, answering questions like 'How do I supply this Mars mission?' However, in many cases we are more interested in evaluating a given architecture such as the Constellation Program architecture, so we must enter the paths through the network by hand.

\section{Simulate and Analyze}

Simulation is the final step before the analysis can begin. The simulation runs through the described logistics architecture and checks for feasibility, ensuring that all demands are indeed met by the transportation paths defined. It also generates the measures of effectiveness for later comparison with other architectures, and produces a series of outputs for analysis. Two types of visualizations are produced, along with a set of detailed data exported to Excel which tracks the movement of supplies and elements at every time step.

\section{SpaceNet Architecture}

This section provides more details on the implementation of the SpaceNet tool. Because the modeling framework was described in detail in Section II, this section focuses on the specific implementation aspects of SpaceNet.

\section{Graphical User Interface}

SpaceNet provides a graphical user interface (GUI); one screen is pictured in Figure 8. The GUI walks the user through all the steps described above. It allows for the definition of surface missions, editing the demand model parameters and generating demand, and the definition of the paths through the network (the GUI screen for definition of paths is shown in Figure 8). The GUI runs within the Matlab environment, and interfaces with the simulation and an Excel-based relational database. The visualization capabilities also depend on the Matlab environment, while the export functions provide data in Excel spreadsheets. 


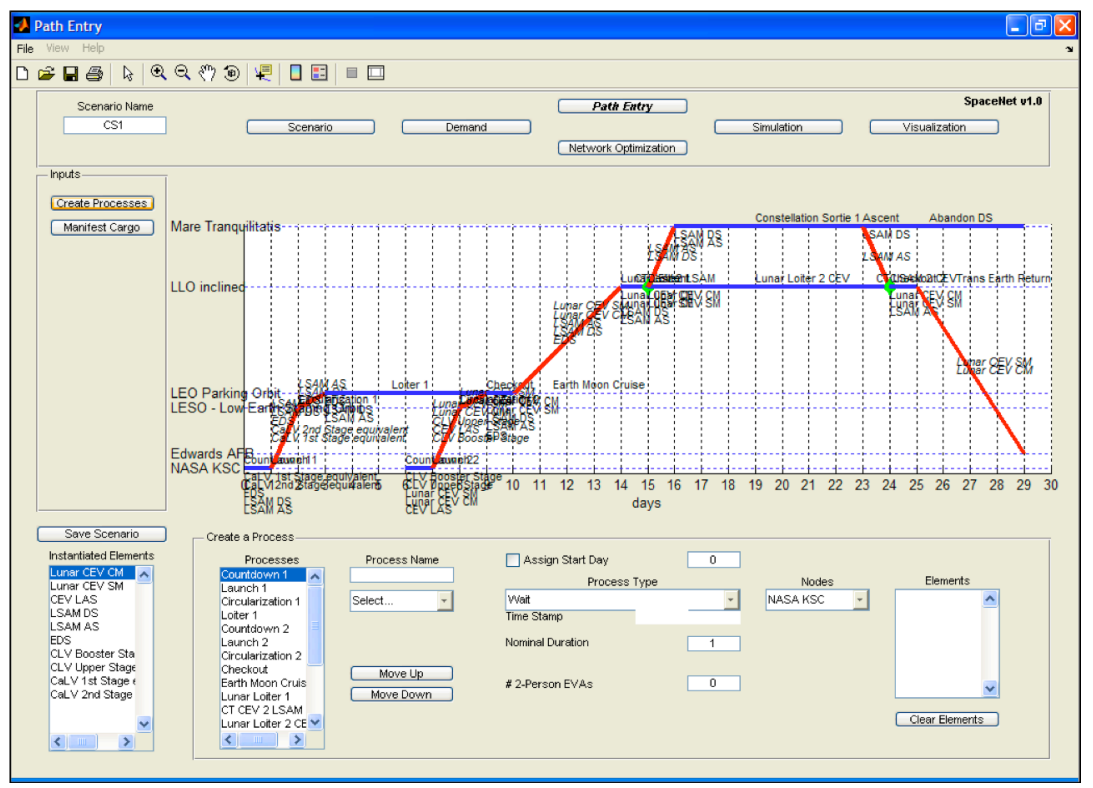

Figure 8. Graphical user interface. One screen from the SpaceNet graphical user interface is shown. The interface for defining paths through the network is shown. Red arcs indicate transport, blue arcs show elements waiting at the same node, and green diamonds represent transfers. Nodes are represented as horizontal dashed lines on the screen, with KSC at the bottom. The screen shows the baseline NASA Constellation EOR-LOR architecture [11].

The GUI has two major functions. First, it provides an interface to the model itself by walking the user through the steps of defining or editing scenarios, and second, it populates the data structures used by the remainder of the model. Thus, it provides the description of each scenario based on user input. This description serves as the input to the SpaceNet simulation.

\section{Simulation}

The SpaceNet simulation takes as input a description of a logistics architecture, and outputs a set of data based on the performance of that scenario. The simulation is also written in Matlab. The simulation carries out the defined processes for the scenario in order. Rather than specifying the times that each element performs actions, each element has a list of actions that are carried out sequentially. This means that the simulation is more event-driven than time-driven. The demand models for crew provisions, crew operations, and spares are called when necessary, such as crew provisions for a process with an element carrying crew. These models calculate the consumption for their respective classes of supply and update the element histories accordingly. The simulation also implements an extensive feasibility check, detecting and reporting such errors as "not enough propellant to complete transport arc" or "crew provisions insufficient", The remaining outputs are discussed below.

\section{Relational Database}

The relational database provides the information architecture for the model. It is discussed extensively in a companion paper [5]. The primary purpose of the database is to store all the information required by the model, such as available element types and their characteristics, nodes and the allowable arcs between them, supply items, etc. Another potential use for the database is real-time operations support; the database is structured so that it can be used to track individual supply items as they move through a real transportation network. Currently, the database is maintained in Excel; however, we are in the process of porting it to a server-based SQL database, which will interface with the same Matlab functions which previously used Excel. The database is thus the main repository for information that is not specific to a single logistics scenario, but rather applies to all of them (a 'library' of available nodes and elements). The scenarios themselves are saved separately by SpaceNet.

\footnotetext{
${ }^{*}$ Specifically, satisfaction of the rocket equation is ensured and residual propellant is computed for all transport arcs.
} 


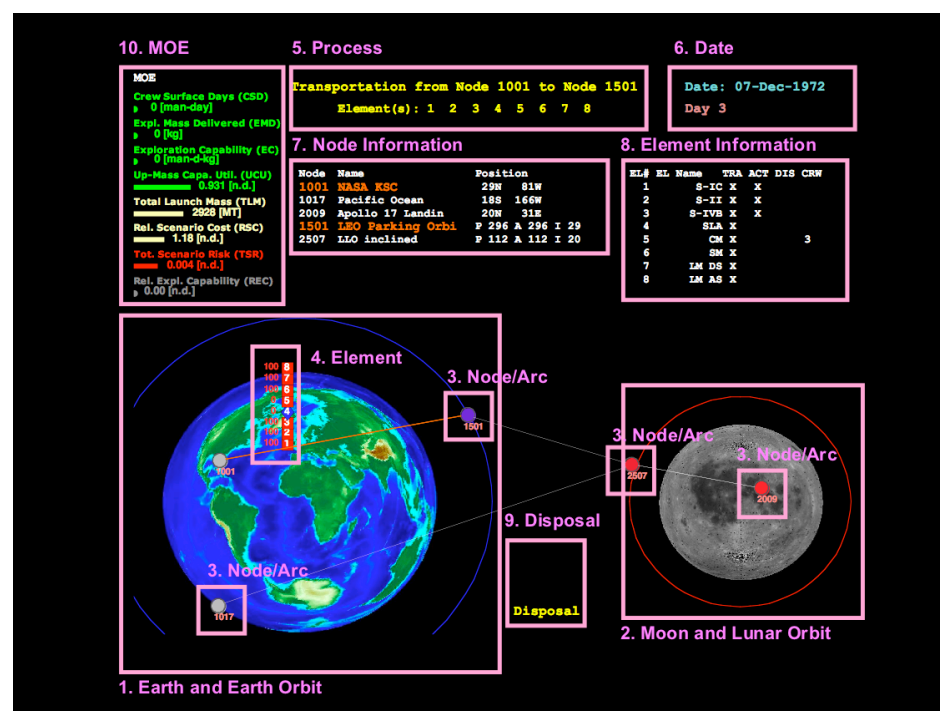

Figure 9. Network visualization. The network animation displays how elements (4) move through the network over time. Nodes and arcs (3) are represented on maps of the Earth and Moon (1) and (2). The date (6) and active process (5) are displayed above, along with information on nodes (7) and active elements (8). Elements disposed (after exhausting fuel) are shown at (9), and the MOEs are displayed (10).

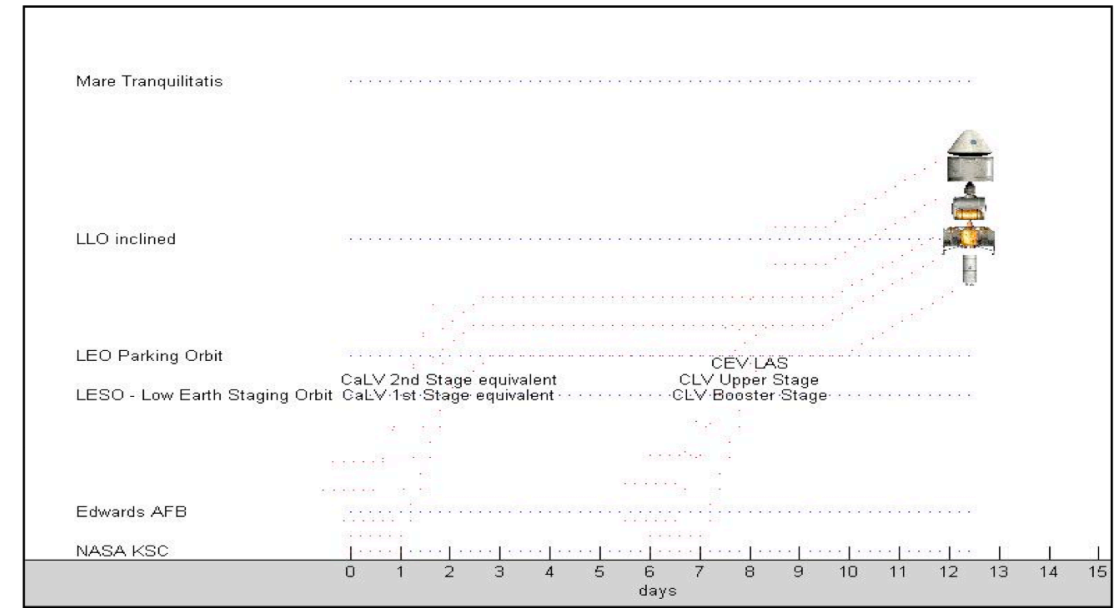

Figure 10. Bat Visualization. The bat visualization displays how elements move through the network over time, on a display with the nodes plotted on the y-axis and time on the x-axis.

\section{Outputs}

SpaceNet generates a number of outputs designed to assist the user in analyzing and comparing logistics architectures. Three types of visualizations are provided, along with an extensive data export capability.

The first type of visualization is a network animation, which shows how elements move through the static network over time. Figure 9 shows the network visualization, which displays the elements as small numbered boxes moving through a network of nodes laid out on maps of the Earth and Moon. Further information is shown in text displays (see figure caption for details). This type of visualization provides an intuitive understanding of the interplanetary transportation network.

The second type of visualization is dubbed the 'bat visualization'. It is an animated version of the common display format in which nodes are plotted vertically along the screen, and time moves along the $\mathrm{x}$-axis. The bat visualization is shown in Figure 10. Again the visualization allows the analyst to understand the logistics architecture and 'catch' inconsistencies and inefficiencies.

The third major output of SpaceNet is a set of detailed time history data in the form of Excel worksheets. This file stores the scenario element history data exported from SpaceNet for easy data interpretation and analysis. The element history data is arranged into six different history tables with the data organized by element, node, or class of 


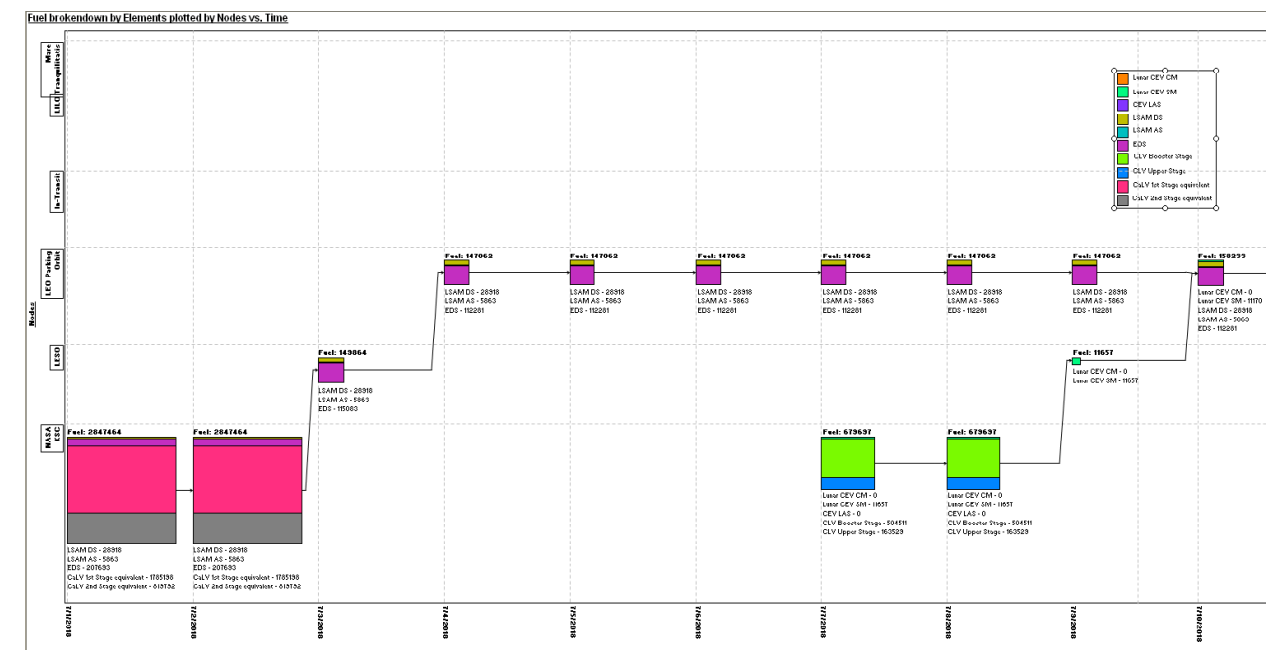

Figure 11. Data plotting capability. Excerpt of fuel history vs. nodes vs. time for EOR-LOR lunar sortie.

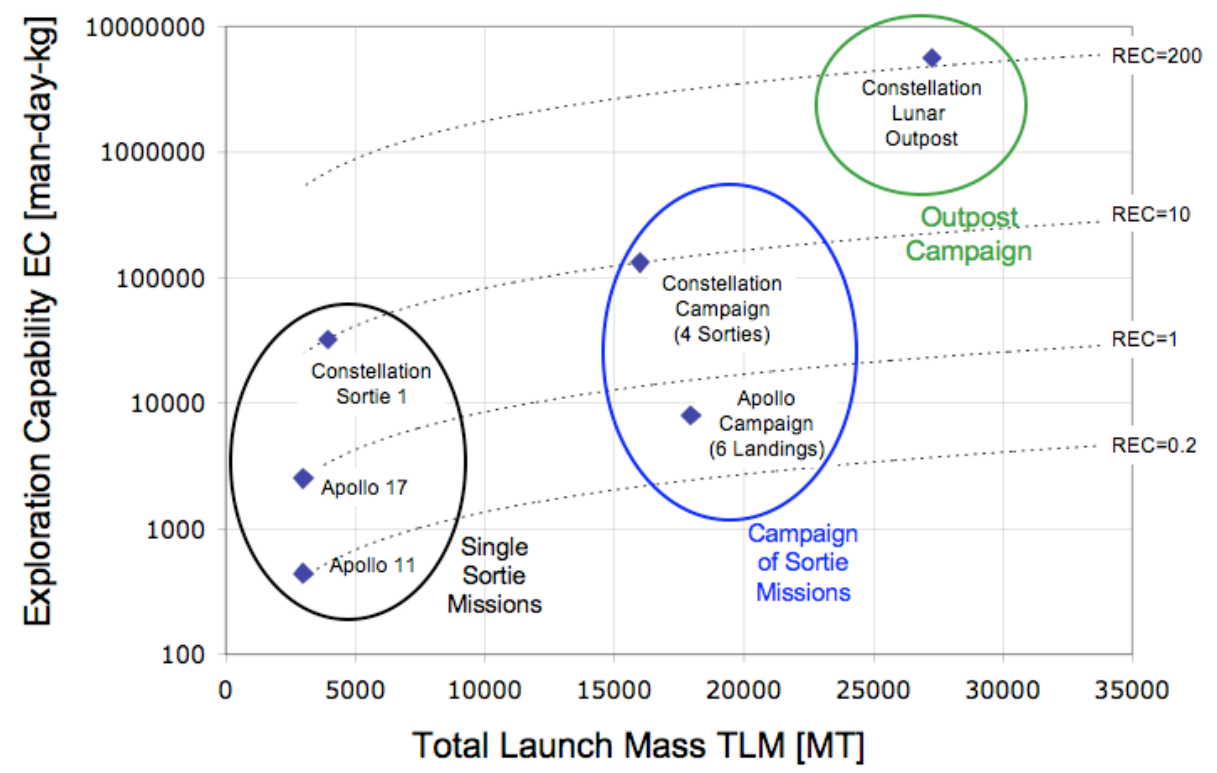

Figure 12. Space logistics trade space. The exploration capability MOE (see Section II) plotted against the total launch mass for various sortie and campaign scenarios modeled in SpaceNet. The advantage of an integrated logistics approach can be seen: both the Constellation and Apollo campaigns are unable to gain exploration capability beyond a multiple of each sortie mission (i.e. same REC), while the lunar outpost buildup shows a greater increase in exploration capability because of its dependence on earlier missions.

supply. The six perspectives allow for examining what supplies were at each node at each point in time, where each element was at each point in time, etc. An additional built-in history plotter can read the data from the history tables and generate mass tracking diagrams. Once created, Excel history files are separate from SpaceNet and can be opened and manipulated with Excel. Figure 11 shows the history of fuel consumption for the base Constellation EOR-LOR architecture [11].

\section{SpaceNet Summary}

SpaceNet is a software tool built to utilize the space logistics modeling framework described in Section II. SpaceNet provides a graphical user interface which allows analysts to describe complex space logistics architectures using the basic concepts of nodes, elements, and supplies. Built-in demand models, a unified database of nodes, vehicles, astrodynamics constraints and supplies, and an optimization capability assist the user in describing various types of supply chains. Such logistics scenarios can then be simulated and assessed for feasibility and performance. 
In summary, SpaceNet models virtually any manned exploration architecture in the Earth-Moon-Mars system, and simulates/tracks the flows of cargo, vehicles, and people. The software evaluates and optimizes logistics and transportation architectures for affordability and sustainability. As such, it supports architecture-level trade studies for space logistics, and can act as an integrated planning and simulation tool.

\section{Results and Conclusions}

\section{A. Results Overview}

A detailed discussion of SpaceNet results is beyond the scope of this paper (see the upcoming publication [10]). Here, we provide a basic overview of the type of results that can be obtained by modeling space logistics architectures. With the modeling framework described herein, we have modeled both single 'sortie' missions and entire campaigns such as the lunar outpost build-up. Specifically, we have modeled the basic Constellation sortie mission, along with Apollo 17. Variations include LEO refueling of Constellation missions and ISRU on the lunar surface. Two major campaigns modeled in SpaceNet are the build-up and operation of the Constellation lunar base (four base pre-deploy missions and the crew/re-supply cycle), and a two-year historical ISS assembly and re-supply scenario.

Figure 12 shows a sample plot from this type of analysis which shows how valuable the logistics modeling approach can be. The plot shows a tradespace with the exploration capability MOE plotted against total launch mass. The Apollo and Constellation sorties and campaigns are plotted in this tradespace. It is clear that the Apollo and Constellation campaigns consisting of a series of sorties (center oval) have exploration capabilities equivalent to multiples of each single campaign. Thus, the relative exploration capability (REC) is the same. However, the lunar outpost mission builds upon the equipment and supplies left by previous missions, so it can gain in relative exploration capability. Such results prove that an early focus on the logistics strategy can help planners to enhance returned value (exploration capability).

\section{B. Summary and Conclusions}

This paper describes in detail a modeling framework for interplanetary supply chain management. We build on the proven techniques of commercial supply chain management to model and evaluate logistics architectures. The framework is built on the basic concepts of nodes, supplies, and elements, tied together into a time-expanded network with a set of processes governing movement through the network. These elements enable coherent technical descriptions of logistics architectures, and a simulation layer provides feasibility assessments and various visual and data reporting outputs for further study by analysts. A set of logistics-related measures of effectiveness are proposed for comparative evaluation of logistics scenarios modeled with this framework.

SpaceNet is a software tool built around this modeling framework. It provides a graphical user interface, built-in demand models, an integrated database with libraries of nodes, vehicles, and supplies, and visualization and Excel reporting capabilities. It has been tested with the Apollo and Constellation scenarios; early results prove its potential to generate unique conclusions on the affordability and efficiency of various types of logistics scenarios.

The modeling framework proposed here (and its current implementation in SpaceNet) enables modeling the flow of crew, cargo, and vehicles through the Earth-Moon-Mars system. The results can support both short- and longterm architectural trade studies, and highlight ways in which space logistics can be improved, enhancing the affordability and robustness of future manned exploration missions.

\section{Future Work}

This project is currently proceeding with a revision of the SpaceNet software and an integration of the optimization capability. A first set of results will be published shortly, and the tool is currently being used to support NASA trade studies. Future plans include additional study of analogous terrestrial supply chains, refinement of the modeling framework for ease of architecture definition, and enhancement to support specific types of trades such as crew time and sparing strategies.

\section{Acknowledgments}

The authors would like to acknowledge the work of Matthew Silver in various aspects of this work, especially the discussion of measures of effectiveness. Additionally, the modeling framework and software described herein was developed by a larger team including Prof. Diego Klabjan, Christine Taylor, Jaemyung Ahn, and many others. 
This work was completed as part of the Interplanetary Supply Chain Management \& Logistics Architectures project financially supported by NASA under contract NNK05OA50C. Prof. Olivier de Weck and Prof. David Simchi-Levi, Massachusetts Institute of Technology, serve as the principal investigators, with Dr. Martin Steele from NASA's Kennedy Space Center as COTR. Co-investigators are Dr. Robert Shishko (JPL) and Mr. Joe Parrish (Payload Systems Inc.).

\section{References}

1. D. Simchi-Levi, P. Kaminsky, E. Simchi-Levi, Designing and Managing the Supply Chain: concepts, strategies and case studies. Second Edition. New York: McGraw-Hill, 2003.

2. C. Heinrich, and D. Simchi-Levi, "Do IT investments really pay off?" Supply Chain Management Review, May/June 2005.

3. E. Gralla, S. Shull, M. Silver, J. Ahn, A. Siddiqi, O. de Weck, "Remote Terrestrial Sites as Operational/Logistics Analogs for Moon/Mars Bases: the Haughton-Mars Project." AIAA 2006-5659, SpaceOps 2006, Rome, Italy, 19-23 Jun 2006.

4. W.A. Evans, O. de Weck, D. Laufer, S. Shull, "Logistics Lessons Learned in NASA Space Flight." NASA/TP2006-214203, 2006.

5. S. Shull, E. Gralla, O. de Weck, R. Shishko, "Future of Asset Management for Human Space Exploration: the Interplanetary Supply Chain Management Database." AIAA 2006-7232, AIAA Space 2006, San Jose, California, 19-21 Sep 2006.

6. C. Taylor, M. Song, D. Klabjan, O. de Weck, and D. Simchi-Levi, "Modeling Interplanetary Logistics: A Mathematical Model for Mission Planning." AIAA 2006-5735, SpaceOps 2006, Rome, Italy, 19-23 Jun 2006.

7. C. Taylor, M. Song, D. Klabjan, O. de Weck, and D. Simchi-Levi, "A Mathematical Model for Interplanetary Logistics." SOLE 2006, Houston, TX, Aug 2006.

8. C. Hulten, "Divisia Index Numbers", Econometrica, Vol. 41, No. 6, Nov 1973.

9. O. de Weck, M. Silver, and R. Shishko, "Measures of Effectiveness for SpaceNet." Unpublished, v3.3, Mar 17, 2006.

10. S. Shull, E. Gralla, and O. de Weck, "A Modeling Framework for Sustainable Space Logistics: Results and Recommendations." accepted at the International Astronautical Congress, Valencia, Spain, Oct 2006.

11. "NASA's Exploration Systems Architecture Study," NASA-TM-2005-214062, Nov 2005. 